\title{
A prospective evaluation of strength and endurance of ankle dorsiflexors-plantar flexors after conservative management of lateral malleolar fractures
}

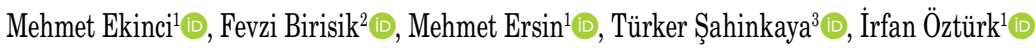 \\ ${ }^{1}$ Department of Orthopaedics and Traumatology, Istanbul University Istanbul Faculty of Medicine, Istanbul, Turkey \\ ${ }^{2}$ Department of Orthopaedics and Traumatology, Istanbul Training and Research Hospital, Istanbul, Turkey \\ ${ }^{3}$ Department of Sports Medicine, Istanbul Medical Faculty, Istanbul, Turkey
}

Received: October 20, 2019 Accepted: May 14, 2020 Published online: September 01, 2021

\begin{abstract}
Objectives: This study aims to evaluate patients with conservatively treated stable lateral malleolar fractures with isokinetic tests.

Patients and methods: Between January 2016 and November 2017, a total of 24 patients (12 males, 12 females; mean age $40.8 \pm 15.0$ years; range, 18 to 68 years) with an isolated stable lateral malleolar fracture treated conservatively with the circular cast were included in this prospective study. Calf circumferences, ankle range of motion (ROM), pain levels, and functional outcomes were recorded. The muscle strengths and endurance of the injured side were compared with the non-injured side. All patients were evaluated by isokinetic test after removal of the cast, and three and six months after the rehabilitation period.

Results: The ROM was found to be lower after removal of the plaster cast, compared to the contralateral ankle. During cast removal, we also found that both dorsiflexor and plantar flexor muscle strength decreased by $25.6 \%$ and $44.7 \%$, respectively, and decreased to $10.3 \%$ and $3.6 \%$ at three months post-rehabilitation. At the end of six months, no statistically significant difference was found between the two sides. In the dorsiflexion-plantar flexion endurance values, $37.8 \%$ and $54.1 \%$ deficit were detected before the rehabilitation protocol, respectively $(\mathrm{p}<0.05)$. At three months, these values decreased to $6.1 \%$ and $13.6 \%$, respectively and the endurances of the injured sides surpassed the non-injured sides $(\mathrm{p}<0.05)$ at six months.

Conclusion: Conservative management of stable isolated lateral malleolar fractures with circular cast causes atrophy and decreases strength-endurance of the calf muscles due to immobilization. These changes are expected to diminish over time and functional outcomes are excellent with a good rehabilitation program.
\end{abstract}

Keywords: Conservative treatment, isokinetic test, lateral malleolar fracture, strength and endurance.

Fractures of the ankle are widespread in daily practice, constituting $9 \%$ of all fractures. ${ }^{[1]}$ Stability of the fracture and preservation of the continuity of the ankle mortise are the main factors to consider while choosing the most optimal treatment. ${ }^{[2,3]}$ Unstable ankle fractures need surgical intervention (open reduction and internal fixation), while stable fractures can be managed conservatively. ${ }^{[4,5]}$ Immobilization of the ankle with plaster and some period of nonweightbearing (NWB) activity are common. These conservative treatments, however, can be challenging and may cause atrophy of the leg muscles, limitation of ankle motions, loss of muscle strength, deep vein thrombosis, and other complications. ${ }^{[6,7]}$

Although muscle weakness and atrophy are predictable and well-known results of immobilization,

Corresponding author: Mehmet Ekinci, MD. Haseki Ĕgitim ve Araştırma Hastanesi Ortopedi ve Travmatoloji Kliniği, 34130 Fatih, İstanbul, Türkiye.

e-mail: dr.ekincimehmet@gmail.com 
changes in the quantitative values of ankle dorsi- and plantar flexion strength and endurance have not been clearly explained, yet. Plantar flexion strength of the ankle after immobilization for ankle fractures has been evaluated in several studies, ${ }^{[8,9]}$ although there is limited evidence on the dorsiflexor strength and ankle endurance, when immobilization is terminated. Moreover, previous studies included non-homogenous patient populations consisting of lateral malleolar, bimalleolar, and trimalleolar fractures. ${ }^{[8,9]}$

In the present study, we aimed to evaluate the changes in ankle dorsi- and plantar flexion strength and endurance in conservatively treated (casting and NWB activity) stable lateral malleolar fractures, and to clarify how muscle strength decreased after immobilization and changed after rehabilitation programs.

\section{PATIENTS AND METHODS}

This prospective study was conducted at Department of Orthopaedics and Traumatology, Istanbul University Istanbul Faculty of Medicine between January 2016 and November 2017. Patients who were referred to our institution and had an isolated stable lateral malleolar fracture type B according to Danis-Weber classification indicated for conservative treatment were screened. We recruited patients with acute isolated stable unilateral lateral malleolar fractures as assessed by the Orthopedic Council Of Istanbul Medical Faculty to be treated conservatively, those aged $>18$ years, having an isolated lateral malleolar fracture (Danis-Weber type B), having no trauma to the another part of the body, medial tenderness, talar shift (suspected deltoid ligament injury), arthrosis of the ankle joint, or history of any previous surgery from lower limbs. Exclusion criteria were as follows: having additional injuries (i.e., deltoid ligament injury, trauma of another part of the body, multi- or polytrauma patients), open fractures, suprasyndesmotic fractures, previous ankle trauma including fractures, previous surgery after fracture displacement during follow-up (i.e., any displacement $>2 \mathrm{~mm}$ in fracture reduction was considered as the loss of reduction), status of unable to walk before the injury, non-compliance to rehabilitation protocol (i.e., advanced dementia), and not willing to participate in the study. Thirtyone patients were included in the study. Seven (22\%) patients who did not comply with the recommended follow-up protocols were excluded. Finally, a total of 24 patients (12 males, 12 females; mean age 40.8 \pm 15.0 years; range, 18 to 68 years) with an isolated stable lateral malleolar fracture treated conservatively with the circular cast were included. A written informed consent was obtained from each patient. The study protocol was approved by the Ethics Committee of Istanbul Medical Faculty (No. 2017/736). The study was conducted in accordance with the principles of the Declaration of Helsinki.

\section{Follow-up program}

The patients were called for fracture follow-up on Day 1, at two, four, and six weeks, and after the removal of the plaster at one week, three and six months for isokinetic tests.

At each visit after the plaster was removed, all patients were assessed for ankle dorsiflexion/plantar flexion with use of a goniometer, standard radiographic assessments, and bilateral calf circumference measurements. At each visit, the patients were asked about pain and at three and six months, and the American Orthopaedic Foot \& Ankle Society (AOFAS) scores and the Foot and Ankle Disability Index (FADI) scores were recorded. The AOFAS scores consist of three main items of pain, function (activity restriction, restriction of walking distance and difficulty on any walking surfaces, walking abnormality, range of ankle flexion plus extension (sagittal) and range of hindfoot inversion plus eversion, ankle-hindfoot stability) and alignment (score $0-100$ ). The FADI is a questionnaire of 26 items including pain level and restriction level of daily activities (score $0-100$ ). The AOFAS evaluates the patient for four weeks, while the FADI measures only the past week. ${ }^{[10,11]}$

All demographic data and Visual Analog Scale (VAS) scores were recorded for each patient's level of pain at the time of arrival to the emergency room. Before circular casting, both calf circumferences were recorded by measuring with a tape from proximal superior third of the distance between the head of the fibula and the lateral malleolus. The patients were called for re-examination of both cast and circulation of the limb the day after plaster was applied, and postplaster pain levels were required using VAS scoring on the first day.

In routine outpatient clinic examinations, fracture healing levels were monitored by radiographs at two-week intervals. After removing the plasters of the patients whose fractures healed and/or who completed six weeks of immobilization in cast, calf circumferences were measured and the ankle dorsiand plantar flexion ROM values were recorded. After plaster removal, malleol-supporting ankle brace 
(Aircast ${ }^{\oplus}$; DJO, Guildford, UK) was applied for the first two weeks and patients were allowed full weightbearing and encouraged to do ankle ROM exercises. The single physiotherapist who arranged ROM and weightbearing exercise programs followed all the patients and met the directives about exercise programs. The patients were also told to discourage the orthosis over the following two weeks.

After the first week of plaster removal, both ankle plantar and dorsiflexors' strength [peak torque (PT) BW\% (body weight)] and endurance \{durability, Total Work Done (BW\%) (TWD)] were measured with a CYBEX 350 isokinetic dynamometer (HUMAC/CYBEX 2009, Stoughton, MA, USA). Measurements of the injured ankle were compared with the non-injured ankle.

\section{Rehabilitation protocol}

After the first isokinetic test, all patients were followed with a standard 10-week rehabilitation program in coordination with the Sports Medicine Department including ankle ROM, strengthening and, proprioception exercises. The patients were called to visit three times per week for compliance with the rehabilitation program and visits were also training sessions. It was a closely supervised rehabilitation program and the patients were also advised to perform exercises at home according to their physical stage in the program. The home-based program included stretching, ROM and strengthening exercises, application of cold packs, and walking periods (20 to $40 \mathrm{~min}$ ). Although the program started immediately following a six-week immobilization period, the patients were followed for the first two weeks with partial weightbearing activity with an ankle brace and allowed only passive ankle ROM (10 to $12 \mathrm{reps} / 3$ to 5 sets) and stretching exercise (5 min) to prevent a possible iatrogenic injury. Passive ROM and stretching exercises were performed under the assistance of a physiotherapist and the force during them was adjusted according to the patients' tolerance. The patients received application of electrotherapy for pain and edema (15 min), and air compression to support limb circulation during the first two weeks of the program (10 $\mathrm{min})$.

A horizontal bike was used for 10 min to warm up. When the ankle ROM was almost complete $(90 \%$ of uninjured limb) and active movements were pain-free, isometric strength and resistance training using elastic resistance bands was started and resistance was gradually increased (5 to $10 \mathrm{sec} / 10$ to 12 repetitions/ 3 to 5 sets). A treadmill was used for ambulation and endurance training. The patients started with $10 \mathrm{~min}$ of ambulation, which was progressively increased by duration (10 to $20 \mathrm{~min}$ ) and speed (4 to $7 \mathrm{~km}$ ). Proprioception exercises were performed while sitting for three weeks. Then, balance and proprioception studies were performed using a balance board and a trampoline with body weight (10 to $30 \mathrm{sec} / 5$ to $10 \mathrm{rep}$ ) (one leg, eyes opened-closed). These studies were initiated on a constant floor and, then, proceeded on mobile boards. The daily program consisted of joint mobilizations, passive stretching, electrotherapy-ice compression for pain relief, and ankle proprioception exercises, if necessary.

\section{Isokinetic test protocol}

Dorsi- and plantar flexion PT (BW\%) and TWD (BW\%) were measured using an isokinetic dynamometer. Ankle dorsi- and plantar flexor strength tests were applied for injured and non-injured ankles. The tests were performed in prone position and the patients were positioned to the exercise chair according to the manual of the manufacturer.

All measurements were performed between 10:00 A.M. and 01:30 P.M. to minimize the effect of daytime hormonal changes, while the patients were being tested. ${ }^{[12,13]}$ The same investigator applied all the test procedures to all patients to provide standardization. The limb dominance was determined considering the limb preference, while kicking a ball. Prior to the test, the patients were given information about both the strength and endurance measurements of the test.

During the test, dorsiflexion-plantar flexion PT force (strength) evaluations were done in three trials and three test repetitions at 30 degrees/sec angular velocity for both ankles. Tests were interrupted for $60 \mathrm{sec}$ for rest. The TWD (endurance) evaluations were made in five trials and 15 repetitions at 120 degrees/sec angular velocity.

\section{Statistical analysis}

Firstly, we analyzed the literature before planning and calculating sample size for our study. There are studies about isokinetic measurements after ankle fractures. ${ }^{[8,9]}$ Schaffer et al. ${ }^{[8]}$ and Stewens et al. ${ }^{\left[{ }^{[9]}\right.}$ had 10 and 20 patients of different fracture types in their studies, respectively. Based on the literature, we planned more than 20 patients for our study. When our measurements were completed for 24 patients with the same type ankle fracture, we performed post-hoc analysis and found $95.8 \%$ study power to detect a significant difference with our sample size. 
Statistical analysis was performed using the Number Cruncher Statistical System (NCSS) version 07.1.21 (NCSS LLC., Kaysville, UT, USA) program by a blinded statistician. Descriptive data were expressed in mean \pm standard deviation (SD), median (min-max) or number and frequency. Repeated measures analysis of variance (ANOVA) with the Bonferroni-corrected post-hoc test was used to assess the significance of changes in normally distributed variables at different time points. The Friedman test with the Dunn-Bonferroni post-hoc test was used to assess the significance of changes in non-normally distributed variables at different time points. Normally distributed variables were analyzed using the paired t-test. Nonnormally distributed variables between the injured and non-injured ankles were compared using the Wilcoxon signed-rank test. A $p$ value of $<0.05$ was considered statistically significant with a type-I and type-II error of $5 \%$ and $20 \%$, respectively.

\section{RESULTS}

All patients completed the study. Baseline demographic characteristics of the patients are shown in Table 1.

The mean VAS score showed a significant improvement from $6.3 \pm 1.8$ before immobilization to $0.7 \pm 1.1$ after cast removal $(p<0.001)$. Changes in the VAS scores were statistically significant on the first day and at plaster removal, but not during the rehabilitation period ( $\mathrm{p}=0.502$ and $\mathrm{p}>0.05$, respectively).

After rehabilitation, the mean AOFAS scores were $94.4 \pm 6.8$ at three months and $95.9 \pm 5.5$ at six months. Change in the AOFAS score was not statistically significant $(\mathrm{p}=0.213$ and $\mathrm{p}>0.05$, respectively). The mean FADI scores were 95.3 \pm 4.9 at three months and $95.7 \pm 5.9$ at six months. There was no statistically significant difference between

\section{TABLE 1}

Baseline demographic characteristics of patients $(n=24)$

\begin{tabular}{|lcc|}
\hline & $\mathrm{n}$ & Mean \pm SD \\
\hline Age (year) & & $40.8 \pm 15.0$ \\
Sex & & \\
$\quad$ Males & 12 & \\
$\quad$ Females & 12 & \\
Weight $(\mathrm{kg})$ & & $72.3 \pm 14.6$ \\
Height $(\mathrm{cm})$ & & $167 \pm 8.35$ \\
BMI $\left(\mathrm{kg} / \mathrm{m}^{2}\right)$ & & $25.8 \pm 4.2$ \\
Dominant/non-dominant side & $15 / 9$ & \\
\hline SD: Standard deviation; BMI: Body Mass Index. & & \\
\hline
\end{tabular}

the three- and six-month FADI scores $(\mathrm{p}=0.626$ and $\mathrm{p}>0.05$, respectively) (Table 2 ).

On the other hand, we found a statistically significant difference in the dorsi- and plantar flexion motion arcs between the injured and non-injured ankles before rehabilitation, and at three and six months. Measurements of injured side limb were significantly lower than those of the non-injured side $(\mathrm{p}=0.001$ and $\mathrm{p}<0.01$, respectively). However, changes in dorsi- and plantar flexion measurements were only statistically significant on injured limbs $(p=0.001$ and $\mathrm{p}<0.01$, respectively), but not on non-injured limbs (Table 3).

The calf circumference of the injured limbs was significantly lower than non-injured side before rehabilitation and at three months $(\mathrm{p}=0.001$ and $\mathrm{p}<0.01$, respectively). However, differences between the calf circumferences gradually decreased at six months, indicating no significant difference (Table 3 ).

Before the rehabilitation period was initiated (at first isokinetic test which was performed one-week post-cast removal and before rehabilitation period was initiated), a significant difference was noted between the injured and healthy ankle dorsiflexion and plantar flexion PT (BW\%) measurements. The PTs of injured limbs were significantly lower than those of the healthy side ( $p=0.001$ and $p<0.01$, respectively). The PTs of the injured ankles were increased by application of the rehabilitation program and PT measurements were similar at three months during follow-up. Deficit of dorsiflexor and plantar flexor muscle strengths were found to be $25.6 \%$ and $44.7 \%$, respectively, when plaster was removed and the loss decreased to $10.3 \%$ and $3.6 \%$, respectively at three months post-rehabilitation. Eventually, dorsiflexor and plantar flexors PTs of the fractured side surpassed those of the healthy ones at isokinetic tests at six months. The differences between dorsiflexion PTs were not statistically significant $(\mathrm{p}=0.017)$.

For endurance, dorsiflexor and plantar flexor TWD (BW\%) for the injured ankle was significantly

\begin{tabular}{|c|c|c|c|}
\hline \multicolumn{4}{|c|}{$\begin{array}{l}\text { TABLE } 2 \\
\text { Functional results of patients }\end{array}$} \\
\hline & $3^{\text {rd }}$ month & $6^{\text {th }}$ month & \\
\hline & Mean \pm SD & Mean \pm SD & $p$ \\
\hline AOFAS & $94.4 \pm 6.8$ & $95.9 \pm 5.5$ & 0.213 \\
\hline FADI & $95.3 \pm 4.9$ & $95.7 \pm 5.9$ & 0.626 \\
\hline
\end{tabular}

SD: Standard deviation; AOFAS: American Orthopaedic Foot \& Ankle Society; FADI: Foot and Ankle Disability Index. 


\begin{tabular}{|c|c|c|c|c|}
\hline \multicolumn{5}{|c|}{$\begin{array}{c}\text { TABLE } 3 \\
\text { Calf circumferences and range of motions of patients }\end{array}$} \\
\hline & & Injured & Non-injured & \multirow[b]{2}{*}{$p$} \\
\hline & & Mean \pm SD & Mean \pm SD & \\
\hline \multirow{3}{*}{ DF peak torque (BW\%) (Nm/kg) } & Pre-rehabilitation & $48.2 \pm 9.8$ & $59.6 \pm 13.5$ & $0.001^{\star \star}$ \\
\hline & Third month & $66.9 \pm 16.7$ & $65.2 \pm 14.8$ & 0.420 \\
\hline & Sixth month & $69.8 \pm 14$ & $65.1 \pm 15.8$ & $0.017^{\star}$ \\
\hline \multirow{3}{*}{$\mathrm{PF}$ peak torque $(\mathrm{BW} \%)(\mathrm{Nm} / \mathrm{kg})$} & Pre-rehabilitation & $58.9 \pm 20.7$ & $99 \pm 28.2$ & $0.001^{\star *}$ \\
\hline & Third month & $115.8 \pm 40.1$ & $125.7 \pm 34.9$ & 0.058 \\
\hline & Sixth month & $131.2 \pm 35.2$ & $127.9 \pm 33.3$ & 0.356 \\
\hline \multirow{3}{*}{ DF total work done (BW\%) (Nm/kg) } & Pre-rehabilitation & $194.1 \pm 79.8$ & $307.8 \pm 113.4$ & $0.001^{\star *}$ \\
\hline & Third month & $332.5 \pm 143.5$ & $341.6 \pm 131.7$ & 0.565 \\
\hline & Sixth month & $374 \pm 112$ & $355.4 \pm 151.2$ & 0.243 \\
\hline \multirow{3}{*}{ PF total work done (BW\%) (Nm/kg) } & Pre-rehabilitation & $205.6 \pm 110.1$ & $408.4 \pm 153.2$ & $0.001^{\star *}$ \\
\hline & Third month & $465.8 \pm 219.8$ & $525.1 \pm 206.7$ & $0.006^{\star *}$ \\
\hline & Sixth month & $552.9 \pm 189.3$ & $566.2 \pm 223$ & 0.657 \\
\hline $\begin{array}{l}\text { SD: Standard deviation; DF: Dorsiflexion; BW } \\
\text { and first measurement after removal of the } \\
\text { Measurement at six months post-rehabilitatior }\end{array}$ & $\begin{array}{l}\text { weight; PF: Plantar } \\
\text { Chird month: Meas } \\
\text { d Samplest test; }{ }^{*} \mathrm{p}^{<}\end{array}$ & Pre-rehabil & At the end of & $\begin{array}{l}\text { nmobiliza } \\
\text { Sixth mo } \\
\text { icant p val }\end{array}$ \\
\hline
\end{tabular}

lower than for non-injured side at the first isokinetic tests (before rehabilitation) $(p=0.001$ and $p<0.01$, respectively). The dorsiflexors TWD of injured ankles improved with application of rehabilitation program and no significant difference was found between the injured and non-injured limb measurements at three months $(p=0.565$ and $p>0.05$, respectively). The patients demonstrated $37.8 \%$ and $54.1 \%$ deficit in dorsiflexion and plantar flexion endurance values at the beginning, respectively, and the loss decreased to $6.2 \%$ and $13.6 \%$ at three months after rehabilitation. The difference was completely closed at six months, when both limbs reached the same level. Even TWD (BW\%) of injured sides surpassed that of non-injured sides. However, TWD of plantar flexors were still lower than non-injured sides at three-months of follow-up $(\mathrm{p}=0.006$ and $\mathrm{p}<0.01$, respectively). While comparing TWD measurements on the injured side and the healthy side at six months after rehabilitation, the dorsiflexor and plantar flexor TWD (BW\%) scores of the injured side were higher than those of the non-injured side, although the TWD measurements were similar for both limbs (DF: $p=0.243$; PF: $p=0.657$ ) (Table 4, Figure 1).

TABLE 4

Peak torque and total work done/body weight $(\mathrm{Nm} / \mathrm{kg})$ values of the ankle dorsi- and plantar flexor muscles

\begin{tabular}{|c|c|c|c|c|c|c|}
\hline & \multicolumn{2}{|c|}{ Calf circumference $(\mathrm{cm})$} & \multicolumn{2}{|c|}{ Dorsiflexion $\left(^{\circ}\right)$} & \multicolumn{2}{|c|}{ Plantarflexion $\left({ }^{\circ}\right)$} \\
\hline & Injured side & Non-injured side & Injured side & Non-injured side & Injured side & Non-injured side \\
\hline & Mean \pm SD & Mean \pm SD & Mean \pm SD & Mean \pm SD & Mean \pm SD & Mean \pm SD \\
\hline Time of injury & $36.8 \pm 3.0$ & $36.8 \pm 3.0$ & & & & \\
\hline Pre-rehabilitation & $34.9 \pm 2.9$ & $36.8 \pm 3.0$ & $19.5 \pm 3.0$ & $23.5 \pm 4.8$ & $18.0 \pm 5.1$ & $27.1 \pm 4.9$ \\
\hline Third month & $36.4 \pm 2.5$ & $37.0 \pm 2.8$ & $24.8 \pm 6.1$ & $26.2 \pm 3.7$ & $28.3 \pm 6.4$ & $29.9 \pm 5.2$ \\
\hline Sixth month & $36.5 \pm 2.8$ & $36.8 \pm 3.0$ & $26.9 \pm 562$ & $26.2 \pm 5.7$ & $32.9 \pm 7.1$ & $32.3 \pm 6.7$ \\
\hline
\end{tabular}


(a)

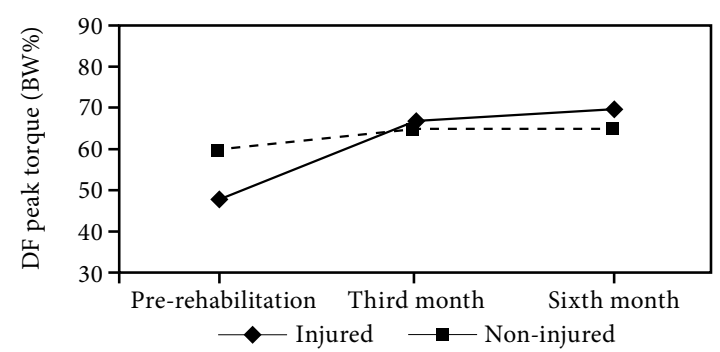

(c)

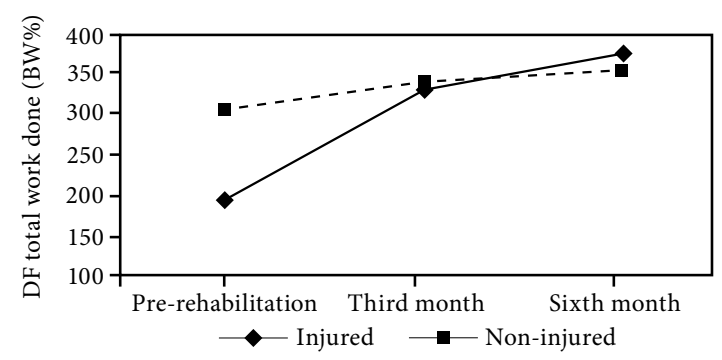

(b)

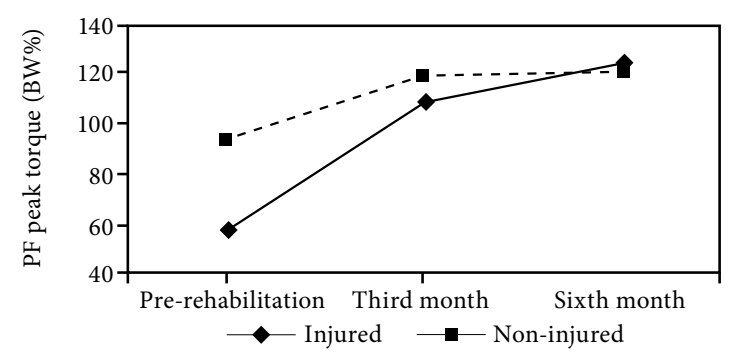

(d)

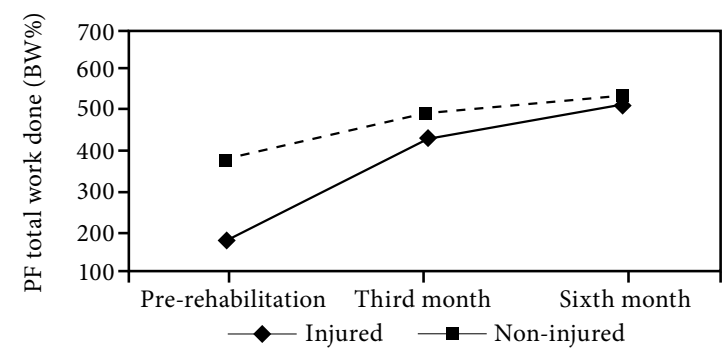

Figure 1. (a-d). Repeated measurements of strength and endurance of both injured and non-injured ankle muscles (DF and PF).

DF: Dorsiflexor; BW: Body weight; PF: Plantar flexor; Pre-rehab: First measurement after removal of the cast; Third month: Measurement at three months post-rehabilitation; Sixth month: Measurement at six months post-rehabilitation.

According to the correlation analysis, a negative correlation was found between the DF TWD and three-month VAS scores $(r=0.449, \mathrm{p}=0.028)$. However, no other correlation was detected between the isokinetic measurements and calf circumferences and VAS scores.

\section{DISCUSSION}

There are many options for conservative management of stable fractures of lateral malleolus. Some comparative studies reported that long-term outcomes of conservatively treated stable fractures were similar for different treatment modalities such as plaster cast, functional brace, or a simple Rest, Ice, Compression, Elevation (RICE) protocol. ${ }^{[14,15]}$ Stewens et al. ${ }^{[9]}$ and Shaffer et al. ${ }^{[8]}$ evaluated the ankle plantar flexor muscle strength and endurance in a non-homogenous ankle fracture group. In contrast to the studies in the literature, our study evaluated a homogeneous patient group prospectively with a standard rehabilitation program and examined the changes in the strength and endurance of the ankle dorsi- and plantar flexor muscle strength with quantitative data. The results demonstrated that muscle atrophy and weakness could be reversible with proper rehabilitation protocol in conservatively treated stable lateral malleolar fractures for a period of three months.
In their study, Stewens et al. ${ }^{[9]}$ evaluated the effect of immobilization on muscle atrophy and muscle strength reduction compared to healthy volunteers. The participants' ankle fractures were treated operatively and immobilized for seven weeks with a short leg cast. Based on the specific moment measurements in the plantar flexor muscles, immobilization with plaster for seven weeks resulted in more than $50 \%$ reduction in the specific strength. The patients received a 10 -week rehabilitation program including passive stretching, proprioception exercises, uphill treadmill walking, and hydraulic plantar flexion machine. Although muscle-specific torque values improved significantly at five weeks of rehabilitation, there was still a $20 \%$ deficit at the end of the 10-week rehabilitation period. Shaffer et al. ${ }^{[8]}$ studied the effects of ankle immobilization for eight weeks after operatively (open reduction-internal fixation)-treated ankle fracture. They permitted weightbearing as tolerated after four weeks of NWB and compared injured ankle with both non-injured ankle and healthy participants. The ankle plantar flexor strength was measured at 1,5 , and 10 weeks after the immobilization was terminated. The plantar flexor PT of the injured ankle was nearly half that of the non-injured limb and approximately $68 \%$ deficit was observed compared to the non-injured individuals. A 10-week rehabilitation program (stretching, motorized 
treadmill, resistance training with hydraulic apparatus) concentrating on strengthening and mobilization was enforced on a three-times-per-week basis for a total of 10 weeks. A significant increase was detected in the plantar flexor PT with five weeks of rehabilitation. At the end of 10 weeks of rehabilitation, the involved ankle had much more torque than the non-injured ankle in the beginning.

Our study results are consistent with the findings of Appell, ${ }^{[16]}$ Duchateau, ${ }^{[17]}$ Stewens et al., ${ }^{[9]}$ and Shaffer et al. ${ }^{[8]}$ Compared to previous studies, we evaluated isokinetic tests at three months after the rehabilitation program was started and found that the differences increased to 10 to $13 \%$. Since the measurements in the Stewens et al.'s ${ }^{[9]}$ and Shaffer et al.'s ${ }^{[8]}$ studies were made at 10 weeks of rehabilitation, it can be speculated that our results are similar, if we consider a two-week difference between our measurement range. Also, Stewens et al..$^{[9]}$ evaluated surgically treated ankle fractures with longer duration of immobilization. Shaffer et al. ${ }^{[8]}$ studied muscle strengths of operated patients by comparing them with uninjured side and healthy volunteers. These injuries were higher-energy trauma than we treated, and more ankle structures were injured in these fractures. However, the deficits of the muscle strengths and improvements during follow-up were similar, despite the use of different rehabilitation programs. However, all of these studies focus on the ankle plantar flexor muscle group. In our study, both the strength and endurance of the plantar flexor and dorsiflexor muscles were evaluated. It is true that the plantar flexor muscle group has a greater importance during walking, but dorsiflexor muscles should not be neglected. This feature of our study contributes to this issue.

In another study, Tropp and Norlin ${ }^{[18]}$ evaluated ankle dorsiflexion strength in different types of operated ankle fractures, dividing them into two groups (immobilized vs. early mobilized) and compared the groups without a standard rehabilitation protocol. Geboers et al. ${ }^{[19]}$ also assessed the ankle dorsiflexion strength in different types of lower limb fractures and compared them with healthy subjects without any specific therapy. Both studies were performed in heterogeneous groups (different types of ankle fractures and lower limb fractures), the plantar flexor muscle group was neglected, a standard physical therapy protocol was not used, and the results were compared with different individuals. The difference in our study and the superiority over these studies is that the patients were prospectively followed with a standard physical therapy protocol in a homogeneous patient group.

In our study, at the end of six months, there was no statistically significant difference, but involved ankles strength and endurance values were higher than the healthy limb. It should be kept in mind that unilateral immobilization of the lower limb may also have a negative effect on the non-injured side. Also, while rehabilitation focuses on the fractured side, the intact side should not be neglected. However, the patients' isokinetic measurements could be taken before applying the plaster to obtain more accurate measurements.

In our study, non-involved limb measurements were not performed before immobilization and they were done six weeks after immobilization. This is the first limitation of our study. Secondly, this study does not have a healthy control group. Also, one group of patients could be followed with only daily activities without any physical therapy after the immobilization period and compared with our participants as a control group. Our third limitation is the relatively small number of patients. Therefore, further large-scale, prospective studies are warranted. Also, the lack of a functional assessment before rehabilitation can be considered as another limitation.

In conclusion, excellent clinical outcomes can be achieved in appropriate patients by treating isolated stable lateral malleolar fractures with conservative methods such as below-knee casts. In addition, effective rehabilitation after removal of plaster can combat the loss of strength and endurance, and help patients return to normal levels within three or six months. Although our findings support what is generally considered to be the common course of action after a common ankle injury, we believe that this study is valuable, as it shows the quantitative results for this path of treatment.

Declaration of conflicting interests

The authors declared no conflicts of interest with respect to the authorship and/or publication of this article.

Funding

The authors received no financial support for the research and/or authorship of this article.

\section{REFERENCES}

1. Court-Brown CM, Caesar B. Epidemiology of adult fractures: A review. Injury 2006;37:691-7.

2. Pakarinen HJ, Flinkkil TE, Ohtonen PP, Ristiniemi JY. Stability criteria for nonoperative ankle fracture management. Foot Ankle Int 2011;32:141-7. 
3. Rammelt S, Grass R, Zwipp H. Ankle fractures. Unfallchirurg 2008;111:421-37.

4. Bauer M, Bergström B, Hemborg A, Sandegård J. Malleolar fractures: nonoperative versus operative treatment. A controlled study. Clin Orthop Relat Res 1985;199:17-27.

5. Bauer M, Jonsson K, Nilsson B. Thirty-year follow-up of ankle fractures. Acta Orthop Scand 1985;56:103-6.

6. Mohtadi N. Injured limbs recover better with early mobilization and functional bracing than with cast immobilization. J Bone Joint Surg [Am] 2005;87:1167.

7. Vioreanu M, Dudeney S, Hurson B, Kelly E, O'Rourke K, Quinlan W. Early mobilization in a removable cast compared with immobilization in a cast after operative treatment of ankle fractures: a prospective randomized study. Foot Ankle Int 2007;28:13-9.

8. Shaffer MA, Okereke E, Esterhai JL Jr, Elliott MA, Walker GA, Yim SH, et al. Effects of immobilization on plantar-flexion torque, fatigue resistance, and functional ability following an ankle fracture. Phys Ther 2000;80:769-80.

9. Stevens JE, Walter GA, Okereke E, Scarborough MT, Esterhai JL, George SZ, et al. Muscle adaptations with immobilization and rehabilitation after ankle fracture. Med Sci Sports Exerc 2004;36:1695-701.

10. Kitaoka HB, Alexander IJ, Adelaar RS, Nunley JA, Myerson MS, Sanders M. Clinical rating systems for the ankle-hindfoot, midfoot, hallux, and lesser toes. Foot Ankle Int 1994;15:349-53.
11. Martin RL, Burdett RG, Irrgang JJ, Martin L, Burdett R, Irrgang J. Development of the Foot and Ankle Disability Index (FADI). J Orthop Sports Phys Ther 1999;29:A32-A3.

12. Hayes LD, Bickerstaff GF, Baker JS. Interactions of cortisol, testosterone, and resistance training: influence of circadian rhythms. Chronobiol Int 2010;27:675-705.

13. Kraemer WJ, Ratamess NA. Hormonal responses and adaptations to resistance exercise and training. Sports Med 2005;35:339-61.

14. Donken CC, Verhofstad MH, Edwards MJ, van Laarhoven CJ. Twenty-one-year follow-up of supination-external rotation type II-IV (OTA type B) ankle fractures: a retrospective cohort study. J Orthop Trauma 2012;26:e108-14.

15. Port AM, McVie JL, Naylor G, Kreibich DN. Comparison of two conservative methods of treating an isolated fracture of the lateral malleolus. J Bone Joint Surg [Br] 1996;78:568-72.

16. Appell HJ. Muscular atrophy following immobilisation. A review. Sports Med 1990;10:42-58.

17. Duchateau J. Bed rest induces neural and contractile adaptations in triceps surae. Med Sci Sports Exerc 1995;27:1581-9.

18. Tropp H, Norlin R. Ankle performance after ankle fracture: a randomized study of early mobilization. Foot Ankle Int 1995;16:79-83.

19. Geboers JF, van Tuijl JH, Seelen HA, Drost MR. Effect of immobilization on ankle dorsiflexion strength. Scand J Rehabil Med 2000;32:66-71. 